\title{
Automatic Performance Model Transformation from UML to C++
}

\author{
Sabri Pllana and Siegfried Benkner \\ University of Vienna \\ Institute of Scientific Computing \\ Nordbergstrasse $15 / \mathrm{C} / 3$ \\ 1090 Vienna, Austria \\ \{pllana,sigi\}@par.univie.ac.at
}

\author{
Fatos Xhafa \\ Polytechnic University of Catalonia \\ Department of Languages and Informatics Systems \\ C/Jordi Girona 1-3 \\ 08034 Barcelona, Spain \\ fatos@1si.upc.edu
}

\author{
Leonard Barolli \\ Fukuoka Institute of Technology \\ Department of Information and Communication Engineering \\ 3-30-1 Wajiro-Higashi, Higashi-ku \\ Fukuoka 811-0295, Japan \\ barolli@fit.ac.jp
}

\begin{abstract}
We address the issue of the development of performance models for programs that may be executed on large-scale computing systems. The commonly used approaches apply non-standard notations for model specification and often require that the software engineer has a thorough understanding of the underlying performance modeling technique. We propose to bridge the gap between the performance modeling and software engineering by incorporating UML. In our approach we aim to permit the graphical specification of performance model in a human-intuitive fashion on one hand, but on the other hand we aim for a machine-efficient model evaluation. The user specifies graphically the performance model using UML. Thereafter, the transformation of the performance model from the human-usable UML representation to the machine-efficient $C++$ representation is done automatically. We describe our methodology and illustrate it with the automatic transformation of a sample performance model.
\end{abstract}

\section{Introduction}

It is impractical and costly to use a large-scale computing system for performance tuning during the program development. Furthermore, in the case of large-scale computing systems the program developer commonly has access to only a part of the computing system resources and for only a limited time. The model-based performance analyzes may be used to overcome these obstacles [16]. Based on the model, the performance can be predicted and design decisions can be influenced without time-consuming modifications of large portions of an implemented program. In the past the performance evaluation of computing systems was a preoccupation of many computer scientists [7, 16]. However, most of approaches $[1,8,5,12,19,9,6]$ for the performance modeling of parallel and distributed programs are of limited use to support performance-oriented software engineering because of the following reasons: (1) the use of a notation that is not based on widely accepted standards, and (2) the requirement that the software engineer has a thorough understanding of the underlying performance modeling technique.

In our approach we aim to bridge the gap between the performance modeling and the software engineering by using the Unified Modeling Language (UML) [2]. We have developed an extension of UML for the domain of performance-oriented parallel and distributed programs [17, 18]. Our UML extension provides a set of UML building blocks that model some of the most important concepts of message passing and shared memory programming paradigms, which can be used to develop models for large and complex parallel and distributed programs. To provide tool support for our approach we have developed the Performance Prophet [15], which is a performance modeling and prediction system. Performance Prophet provides a UML based graphical user interface, which alleviates the problem of specification and modification of the performance model. In the context of Performance Prophet we aim to permit the 
graphical specification of performance model in a humanintuitive fashion on one hand, but on the other hand we aim for a machine-efficient model evaluation. The user specifies graphically the performance model using UML. Afterwards, Performance Prophet automatically transforms the performance model from UML to $\mathrm{C}++$ and evaluates it by simulation.

In this paper we describe our methodology for automatic transformation of performance models from UML to $\mathrm{C}++$. We show how we may develop a UML-based performance model for a given program code, and thereafter we explain how the UML representation of the performance model is transformed to the corresponding $\mathrm{C}++$ representation. Furthermore, we present and explain our algorithm for the performance model transformation from UML to $\mathrm{C}++$, which is implemented in the Performance Prophet. We demonstrate our methodology with the transformation of a sample performance model using the Performance Prophet.

The rest of this paper is organized as follows. Section 2 describes how we customized the UML for performance modeling and outlines the architecture of the Performance Prophet. Our performance model transformation methodology is presented in Section 3. Section 4 exemplifies the model transformation using the Performance Prophet. Finally, Section 5 concludes the paper and briefly describes the future work.

\section{Preliminaries}

In this section we describe our approach for customization of the UML for performance modeling of parallel and distributed programs and give an overview of the architecture of Performance Prophet.

\subsection{UML-Based Performance Modeling}

UML $[2,13]$ is a graphical language that is primarily used for visualizing, specifying, and documenting the software-intensive systems. In order to make possible the modeling of different types of systems, UML modeling elements are defined in UML specification in an abstract manner without conceptual connection with a particular domain. For instance, the UML specification defines the modeling element Action as follows: "an action is the fundamental unit of behavior specification" [13]. Such an abstract definition allows us to use an action to model various kinds of behavior such as addition of two numbers in a computer system, or acceleration of a vehicular system. However, too generic semantics of UML modeling elements may present an obstacle for using UML in a specific domain. For this reason, UML specification defines the mechanisms for specializing semantics of modeling elements for a particular domain. UML extension mechanisms include stereotypes, tagged values, and constraints.

The UML may be extended by defining new modeling elements, stereotypes, based on existing elements, base classes (i.e. metaclasses). A stereotype is defined as a subclass of an existing UML metaclass, with the associated tagged values (i.e. metaattributes) and constraints. Stereotypes are notated by the stereotype name enclosed in guillemets <<StereotypeName $>$, or by a specific graphic icon. Stereotypes may improve the readability of models by distinguishing modeling elements of the same shape with different stereotype names.

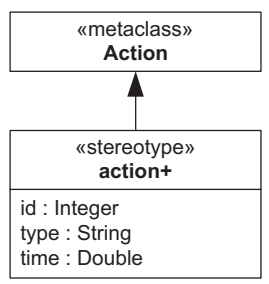

(a) Definition

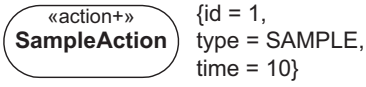

(b) Usage

\section{Figure 1. Definition and usage of the stereo-} type $<<$ action $+>>$.

Figure 1(a) depicts the definition of stereotype $<<$ action $+>>$ based on the UML metaclass Action. The list of tag definitions includes id, type, and time. Tag id can be used to uniquely identify the modeling element $<<$ action+>>; tag type specifies the type of $<<$ action $+>>$, and tag time the time spent to complete $<<$ action $+>>$. We are using $<<$ action $+>>$ (see example in Figure 1(b)) to model various types of singleentry single-exit code regions. Commonly we use tags to describe performance relevant information, such as the estimated or the measured execution time (see the tag time in Figure 1(b)). The set of tag definitions is not limited to those shown in Figure 1(a), but it can be arbitrarily extended to meet the modeling objective. In this manner we have extended the UML for performance modeling of parallel and distributed programs $[17,18]$.

\subsection{Performance Prophet}

Performance Prophet [15] is a performance modeling and prediction system for parallel and distributed computing systems. The architecture of Performance Prophet is depicted in Figure 2. The main components of Performance Prophet are Teuta and Performance Estimator. Teuta is a platform independent tool for graphical modeling of parallel and distributed programs. The role of Performance Estimator, in the context of Performance Prophet, is to estimate the performance of a program on a computing machine. 


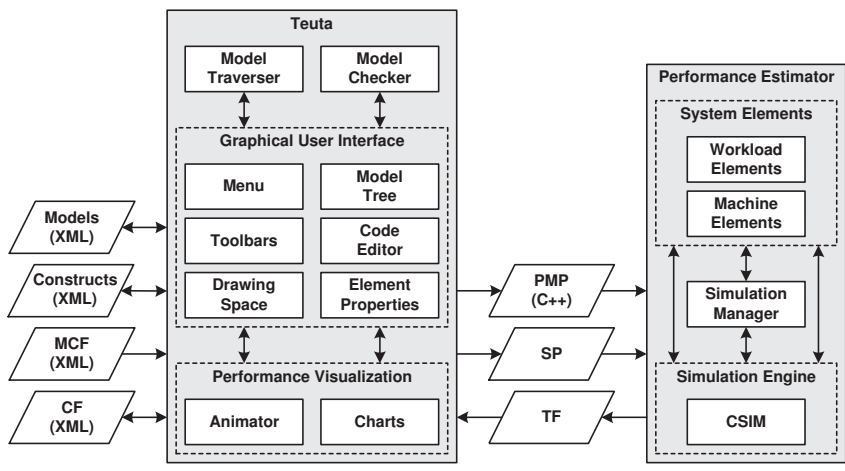

Figure 2. The architecture of Performance Prophet. Abbreviations: Model Checking File (MCF), Configuration File (CF), Performance Model of Program (PMP), System Parameters (SP), Trace File (TF).

Teuta comprises the following parts: Model Checker, Model Traverser, Graphical User Interface (GUI), and the components for Performance Visualization (see Figure 2). The GUI of Teuta is used for the development of performance model based on the UML [13]. The Model Checker is used to verify whether the model conforms to the UML specification. The Model Traverser is used for generation of different model representations (XML and $\mathrm{C}++$ ). The Performance Visualization components are used for visualization of the performance results.

Element MCF indicates the XML file, which is used for the model checking. The XML files that are used for the configuration of Teuta are indicated with the element CF.

The Performance Estimator estimates the performance of a parallel and distributed program on a target computer architecture. As input for the Performance Estimator serve the program model and architectural parameters that are specified in Teuta. The Performance Estimator generates automatically the machine model based on the specified architectural parameters. The program model is integrated with the machine model to create the model of the whole computer system. The Performance Estimator evaluates the integrated model of computing system and generates the corresponding performance results.

The communication between Teuta and the Performance Estimator is done via elements PMP, SP and TF. Element PMP indicates the $\mathrm{C}++$ representation of the program's performance model. PMP is generated by Teuta and serves as input information for the Performance Estimator. Element SP indicates a set of system parameters. The parameters of system include the number of computational nodes, the number of processors per node, the number of processes, and the number of threads. The Performance Estimator uses
SP for building the model of system, whose performance is estimated. Element TF represents the trace file, which is generated by the Performance Estimator as a result of the performance evaluation. Teuta uses $\mathrm{TF}$ for the visualization of performance results.

\section{Methodology}

In this section we describe conceptually the transition: (1) from the program code to the UML based performance model, and (2) from the UML representation to the $\mathrm{C}++$ representation of performance model. Thereafter, we present our algorithm that takes as input the UML representation, and automatically generates the $\mathrm{C}++$ representation of the performance model.

Commonly, scientific programs are written in imperative languages such as Fortran or $C$. This type of programs is executed on parallel and distributed computing systems, which may consist of multiple nodes (each node may have multiple processors), in order to solve large problems or to reduce the time to solution for a single problem [4]. The MPI $[20,11]$ is usually used to express the inter-node parallelism, whereas OpenMP $[3,14]$ is used to express the intra-node parallelism. We have identified that UML activity diagrams are suitable for modeling scientific imperative programs [18]. Therefore, we usually model a scientific program with one or more activity diagrams. Activity diagrams may be annotated with performance-relevant information. For instance, cost functions that model the execution time of program actions may be associated with ActionNodes of the activity diagram.

During the process of performance modeling are considered only the code blocks that strongly influence the overall performance of program. We may identify, for an existing program, code blocks that determine the overall program performance by using a profiling tool.

Figure 3(a) shows a code block of a Fortran program. This code block is known as kernel 6 of the Livermore Fortran kernels [10]. Since the performance of a scientific program is strongly influenced by loops, it is important to consider loops during the development of performance model. Figure 3(b) shows the UML model of kernel 6, which is a fragment of an activity diagram. But, this detailed UML representation of the kernel 6 is not necessary, since we are interested on the rough performance estimation. Therefore, we model the performance of the kernel 6, that is depicted in Figure 3(a), with the action Kernel6, which is an instance of the stereotype action+ (see Figure 3(c)). The associated cost function $F_{K 6}(\ldots)$ models the execution time $T_{K 6}$ of kernel 6.

The UML based representation of performance model of the kernel 6, that is depicted in Figure 3(c), is simple and intuitive. We have developed this graphical representa- 


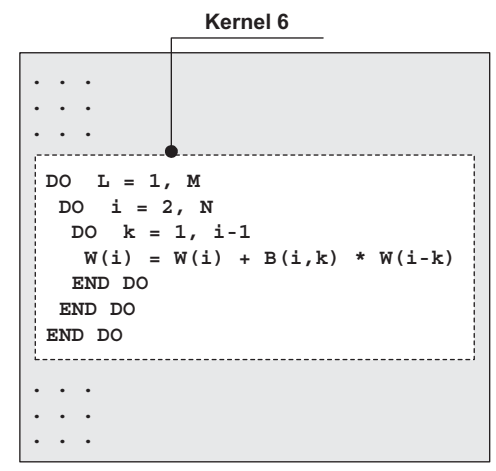

(a)

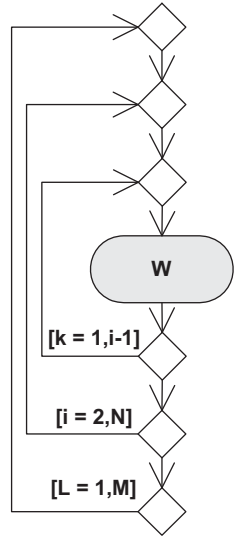

(b) (c)

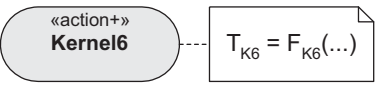

Figure 3. From the program code to the UML based performance model.

tion to streamline the specification process of performance models. However, while the UML representation is suitable as human-usable notation for performance model specification, it is not adequate for an efficient model evaluation. Therefore, we need to transform the UML representation to a form that is suitable for evaluation.

Figure 4 depicts an example of transition from the UML representation to the $\mathrm{C}++$ representation of performance model. For the illustration of this transformation process serves the model of kernel 6 that we introduced in Figure 3 . We use the stereotype action+ to represent a code block of a program. In Figure 4(a) the action Kernel6, which is an instance of stereotype action+, represents the kernel 6. For the modeling element action+ we have defined the corresponding class ActionPlus (see Figure 4(b)). In the context of Performance Prophet, the class ActionPlus is implemented as a $\mathrm{C}++$ class. The properties of modeling element action+ are mapped to properties of the class ActionPlus. The performance behavior of the modeling element action+ is defined in the method execute() of the

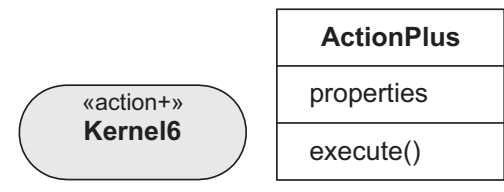

(a)

(b)

Actionplus kernel6(...); kernel6.execute (...,FK6 (...));

(c)

\section{Figure 4. From the UML representation to the $\mathrm{C}++$ representation of performance model.}

class ActionPlus. Figure 4(c) depicts the textual representation of the model of kernel 6 . We may observe that the name of the instance of modeling element (in our example Kernel6) is mapped to the name of the instance of class ActionPlus (in our example kernel6).

Figure 5 depicts our algorithm for the automatic model transformation from UML to $\mathrm{C}++$ representation. As input serves the UML model of a program. The algorithm generates $\mathrm{C}++$ representation of the model. The UML model, with its diagrams and modeling elements, forms a tree data structure. During the model transformation process the tree is programmatically traversed, which makes possible to visit each modeling element and read its properties (see Figure 6). Lines 1-8 of the algorithm determine the performance relevant modeling elements of the UML model based on the element's property stereotype name. For instance, modeling elements with the stereotype name action+ are used to model the performance of sequential code blocks. In the $\mathrm{C}++$ model representation are included the global variables, cost functions, and the model structure (that is performance modeling elements and their flow). Lines 9-12 of the algorithm are responsible for generation of $\mathrm{C}++$ representation of the global variables. Lines 13-18 generate C++ representation of the cost functions (for instance, double FA1 ( ) $\{\ldots\}_{i}$ ). The model structure is defined in the lines 19-35. If there are local variables defined in the UML model, then their $\mathrm{C}++$ representation is generated in the lines 20-23. Lines 24-28 declare the performance modeling elements (for instance, ActionPlus $A 1(\ldots)$;). In the lines $25-35$ it is defined the execution flow of modeling elements. The algorithm generates the $\mathrm{C}++$ code that for each performance modeling element invokes its execute() method (for instance, Al . execute (uid, pid, tid, FA1 ()) ; ). The execution order of performance modeling elements is in accordance with the specified flow in the UML model.

Figure 6 shows the UML communication diagram of the model traversing procedure, which provides the possibility 


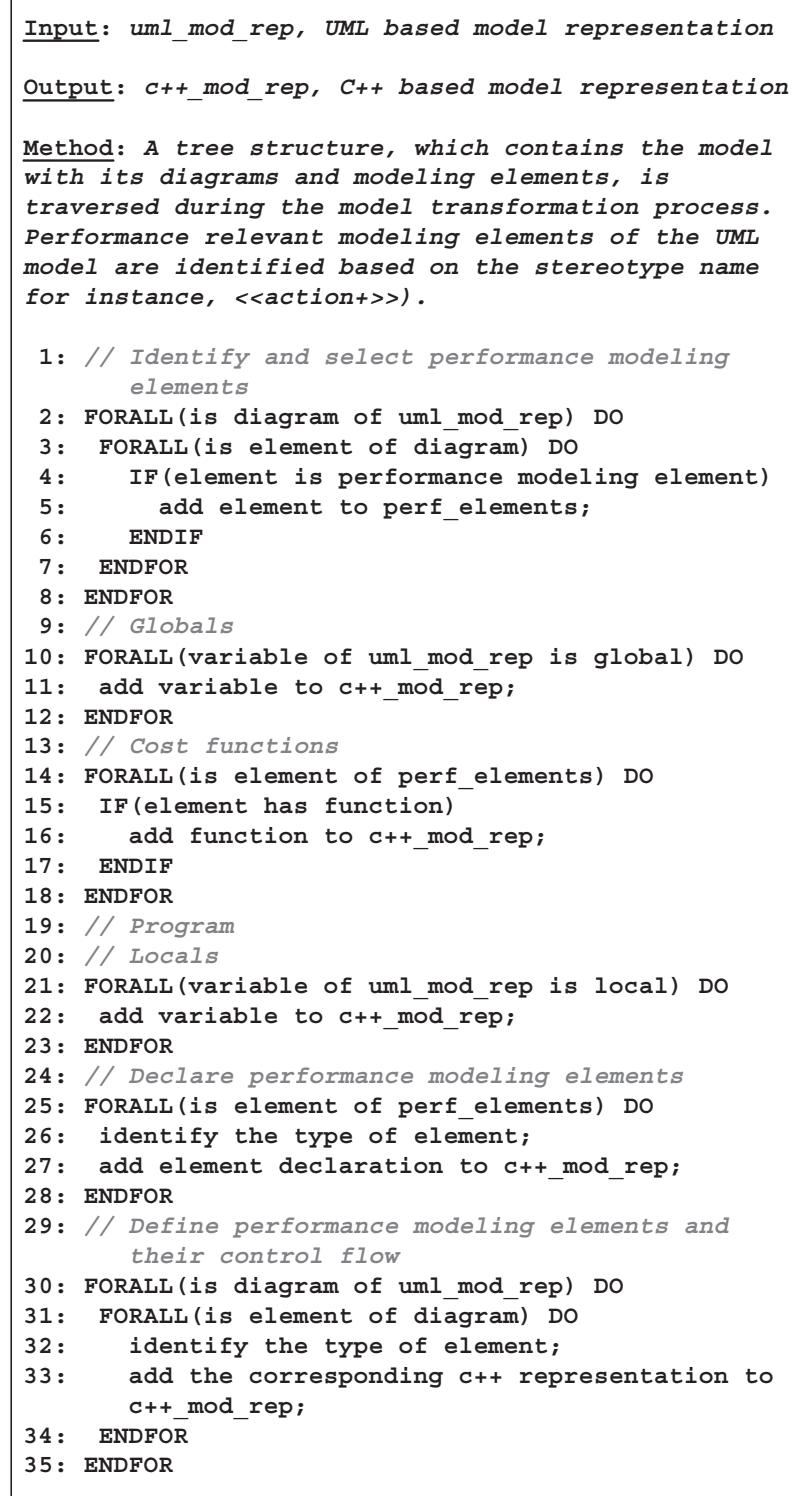

Figure 5. The algorithm for model transformation from UML to $\mathrm{C}++$.

to walk programmatically through the model, to visit each modeling element, and to access its properties. We use the model traversing for the generation of various model representations. Model traversing involves three entities: the Traverser, the Navigator and the ContentHandler. During the model traversing procedure, first, the Traverser sends the navigation command to the Navigator. Then, the Traverser obtains the current element $c e$ from the Navigator. Finally, the Traverser asks the ContentHandler to visit the element $c e$ and generate the corresponding code.

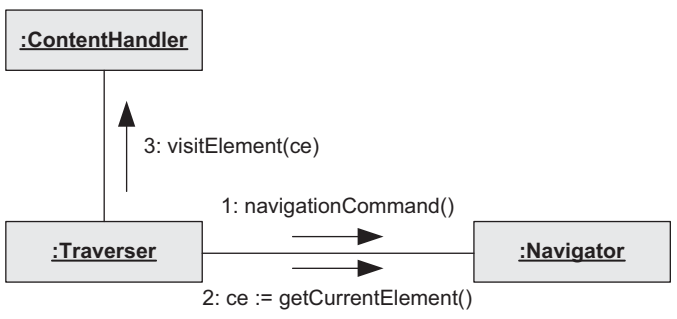

\section{Figure 6. The UML communication diagram of the Performance Prophet model traverser.}

The Navigator, the Traverser, and the ContentHandler are independent of each other in the sense that they only communicate via well-defined interfaces (see Figure 6). Therefore, each implementation of one of these components can be combined with any implementation of the other two components. Performance Prophet provides the necessary interfaces and base classes and default implementations of the Navigator, Traverser and ContentHandler. Commonly, the extension of Performance Prophet for the generation of a specific model representation involves only a specific implementation of the ContentHandler interface.

In the following section we illustrate our methodology with an example using the Performance Prophet.

\section{Example}

Figure 7 depicts the process of UML based specification of a sample performance model for a hypothetical program. The user specifies the type of performance modeling elements and their flow. Furthermore, the user may associate a code fragment and a cost function to each performance modeling element. Each performance modeling element corresponds to a code block of a hypothetical program, whose performance is modeled. The example in Figure 7(a) illustrates the hierarchical modeling capabilities of Performance Prophet. On the left hand side of Figure 7(a) is depicted the main activity diagram, which comprises a set of instances of stereotypes action+ and activity+. After the execution of action $A l$ is completed, based on the value of variable $G V$, it is decided whether to execute the activity $S A$ or the action A2. While an action is not further decomposed into other elements, an activity contains a set of elements. The content of an activity is described with an activity diagram. The content of activity $S A$, which is an instance of stereotype activity+, is depicted in the undocked diagram $S A$ in Figure 7(a). Activity $S A$ comprises performance modeling elements $S A 1$ and $S A 2$.

It is possible to associate global and local variables to the model. The name and the type of global and local variables may be specified as properties of the model. On the 


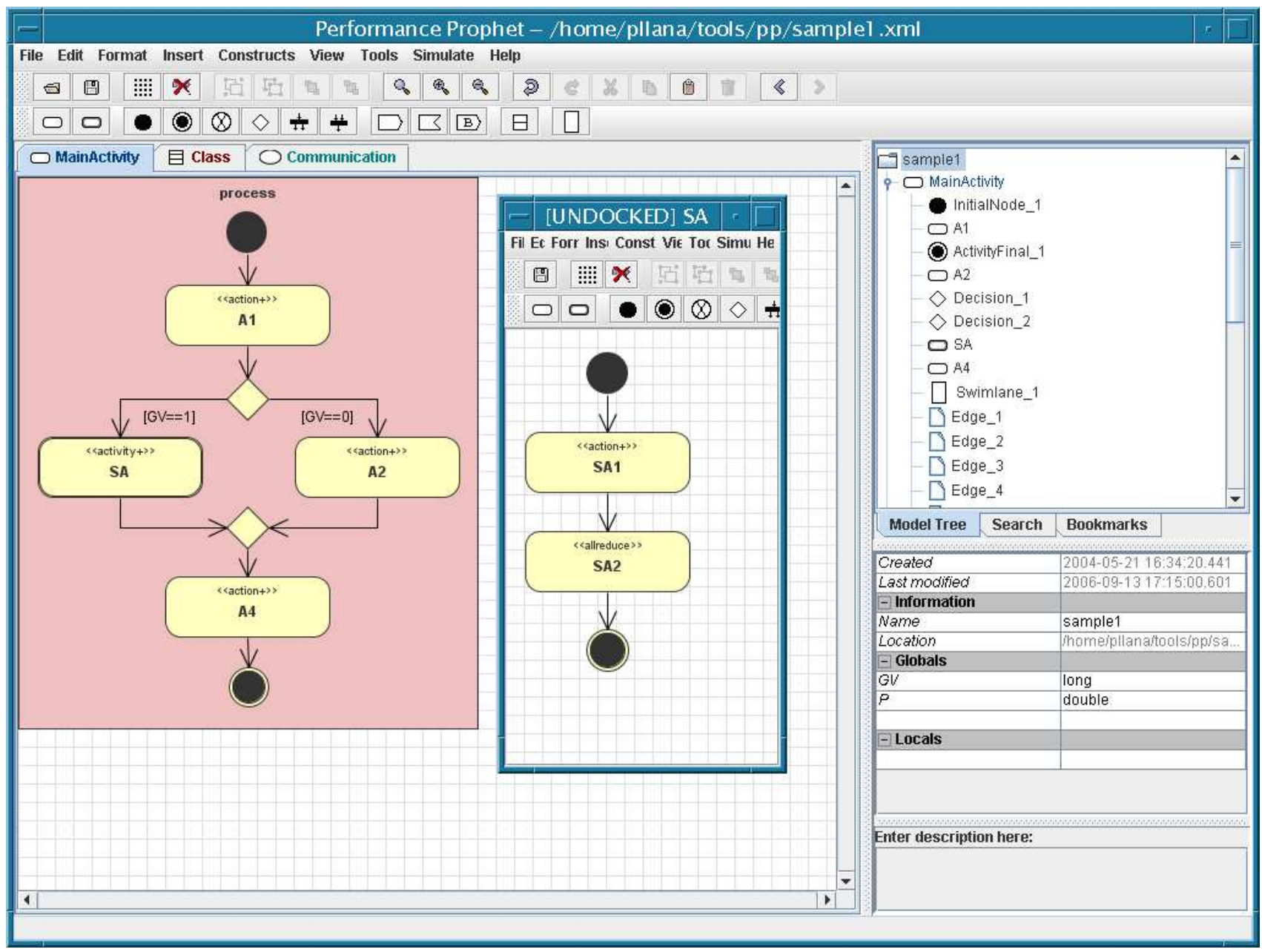

(a) The performance modeling elements and their flow



(b) Code fragment association

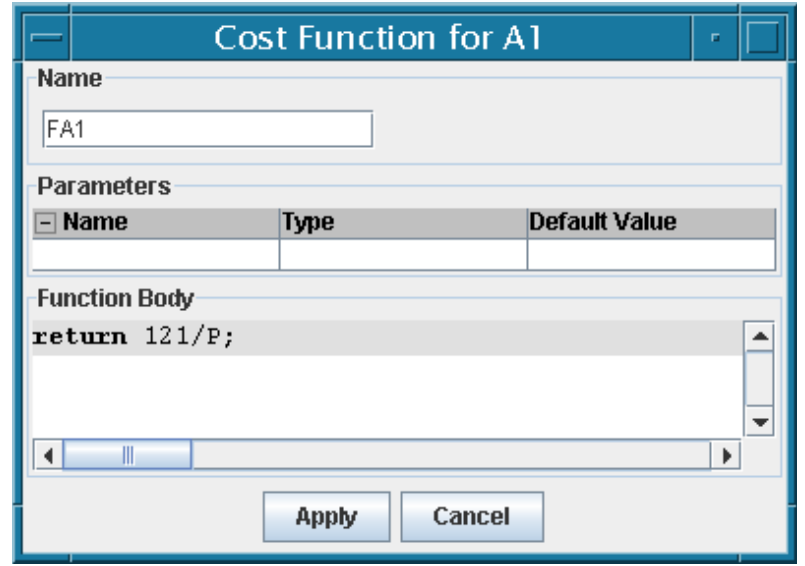

(c) Cost function association

Figure 7. The UML based specification of a sample model. 
right-down corner of Figure 7(a), in the list of properties of sample model, we may observe that variables $G V$ and $P$ are specified as global variables of the model. We are aware that this sample model could be expressed without the use of global variables, but nevertheless we opted for this solution for illustration purposes.

Figure 7(b) depicts an example of the code association to a performance modeling element. This feature can be used to complement $\mathrm{C}++$ representation of the performance model. In this example we have associated a code fragment to the performance modeling element $A l$, which assigns values to global variables $G V$ and $P$.

Figure 7(c) depicts an example of the association of a cost function to a performance modeling element. A cost function models the execution time of the code block that is represented by the performance modeling element. A cost function may use local or global variables as parameters. Moreover, a cost function may be composed using other functions that are defined in the performance model. In this example we have associated a simple parameterized cost function to the performance modeling element $A l$.

Figure 8 depicts the $\mathrm{C}++$ representation of the sample model, which is automatically generated by the Performance Prophet based on the UML representation. The model transformation from UML to $\mathrm{C}++$ representation is based on the algorithm that we presented in Figure 5.

Figure 8(a) depicts an excerpt of the C++ model representation that includes two code sections: (1) global variables, and (2) cost functions. Lines 24-25 declare the global variables $G V$ and $P$. In our sample model the variable $G V$ is used to make the decision whether to execute activity $S A$ or action $A 2$ (see Figure 7(a)). The variable $P$ is used as a parameter of cost functions. We may observe that, in this example, for each performance modeling element $\{A 1, A 2, A 4, S A 1, S A 2\}$ it is defined a cost function $\{F A 1, F A 2, F A 4, F S A 1, F S A 2\}$ (code lines 31-54). As parameters of cost functions may be used the properties of system components (such as number of processors, or the ID of process). For instance, the cost function FSA2 takes pid as a parameter, which is the process ID. Please note that the cost functions presented here serve the purpose of illustration of various forms of expressing cost functions, and that these cost functions are not derived from a real-world program.

Figure 8(b) depicts the declaration of performance modeling elements and their execution flow. Lines 64-68 declare the performance modeling elements $\{A 1, A 2, A 4, S A 1, S A 2\}$. We may observe that the C++ code that represents activity $S A$ (lines 79-82) is nested within the C++ code of the main activity (lines 71-89). Lines $72-75$ represent the code that is associated with the element $A I$ (the code association is depicted in Figure 7(b)). A performance modeling element is executed by invoking



(a) Global variables and cost functions

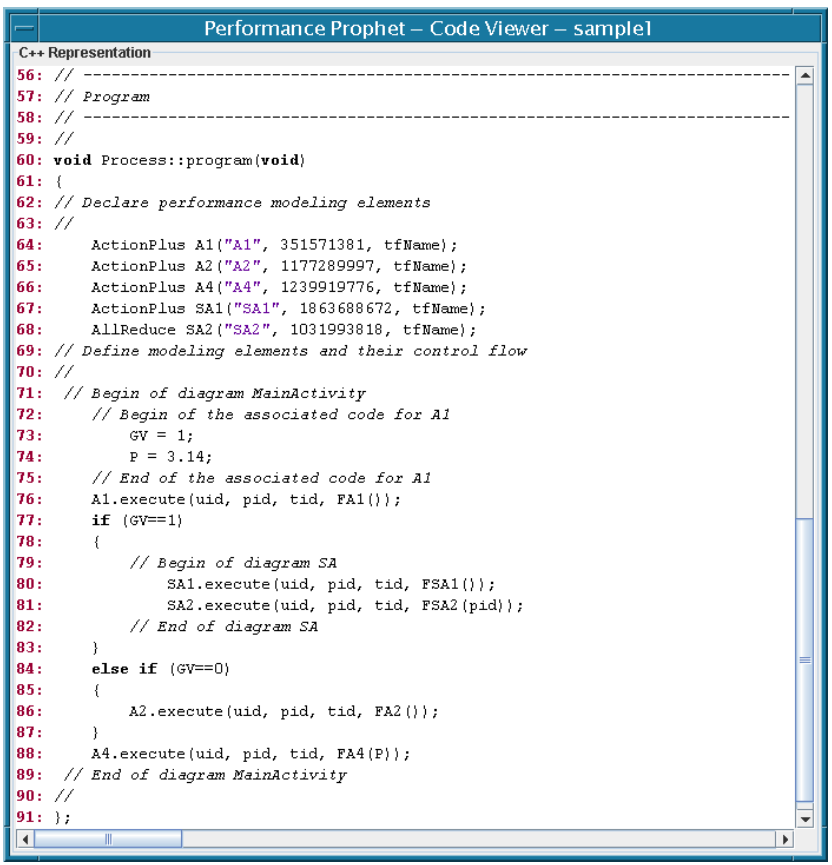

(b) Execution flow of performance modeling elements

Figure 8. The $\mathrm{C}++$ representation of the sample model.

its execute() method. The execution of a performance modeling element models the performance behavior of a code 
block during the program execution. Each performance modeling element corresponds to a code block of the program, whose performance is modeled. For instance, the line 76 in Figure 8(b) executes the performance modeling element $A 1$. We may observe that one of the parameters of method execute() is the name of the cost function FAl that is associated with the element $A 1$. The branch control flow of the UML model representation (see Figure 7(a)) is mapped to the if-else-if statement in $\mathrm{C}++$ model representation (lines 77-87).

The $\mathrm{C}++$ representation that is presented in Figure 8 is used as input for the Performance Estimator.

\section{Conclusions}

In this paper we have described our methodology for the development of performance models of programs. Our approach supports the graphical specification of performance models in a human-intuitive fashion on one hand, and on the other hand is amenable to the machine-efficient model evaluation. The model transformation, from the graphical human-intuitive form (that is, UML representation), to the form that can be efficiently evaluated by machine (that is, $\mathrm{C}++$ representation), is performed automatically. We have demonstrated our methodology with the transformation of a sample performance model using the Performance Prophet modeling system.

In future we plan to extend our approach to enable the automatic generation of the program code based on the UML model.

\section{References}

[1] V. Adve, R. Bagrodia, J. Browne, E. Deelman, A. Dube, E. Houstis, J. Rice, R. Sakellariou, D. Sundaram-Stukel, P. Teller, and M. Vernon. POEMS: End-to-End Performance Design of Large Parallel Adaptive Computational Systems. IEEE Transactions on Software Engineering, 26:1027-1048, November 2000.

[2] G. Booch, J. Rumbaugh, and I. Jacobson. The Unified Modeling Language User Guide, Second Edition. Addison Wesley Professional, 2005.

[3] L. Dagum and R. Menon. OpenMP: An Industry-Standard API for Shared-Memory Programming. IEEE Computational Science and Engineering, 5(1):46-55, Jan. /Mar. 1998.

[4] S. Graham, M. Snir, and C. Patterson. Getting Up to Speed: The Future of Supercomputing. The National Academies Press, 2004.

[5] D. Grove and P. Coddington. Performance Modeling and Evaluation of High-Performance Parallel and Distributed Systems. Performance Evaluation, 60(1-4):165-187, May 2005.
[6] C. Hughes, V. Pai, P. Ranganathan, and S. Adve. RSIM: Simulating Shared-Memory Multiprocessors with ILP Processors. IEEE Computer, 35(2):40-49, February 2002.

[7] H. Karatza. Applied System Simulation: Methodologies and Applications, chapter Simulation of Parallel and Distributed Systems Scheduling, Concepts, Issues and Approaches. Springer, 2003.

[8] D. Kerbyson, A. Hoisie, and H. Wasserman. Use of Predictive Performance Modeling During Large-Scale System Installation. Parallel Processing Letters, 15(4), December 2005.

[9] D. Kvasnicka, H. Hlavacs, and C. Ueberhuber. Simulating Parallel Program Performance with CLUE. In International Symposium on Performance Evaluation of Computer and Telecommunication Systems (SPECTS), pages 140-149, Orlando, Florida, USA, July 2001. The Society for Modeling and Simulation International.

[10] F. H. McMahon. The Livermore Fortran Kernels: A Computer Test of the Numerical Performance Range. Technical Report UCRL-53745, Lawrence Livermore National Laboratory, Livermore, California, December 1986.

[11] Message Passing Interface Forum (MPIF). http://www.mpiforum.org/.

[12] G. Nudd, D. Kerbyson, E. Papaefstathiou, S. Perry, J. Harper, and D. Wilcox. PACE - A Toolset for the Performance Prediction of Parallel and Distributed Systems. International Journal of High Performance Computing Applications, 14(3):228-251, 2000.

[13] Object Management Group (OMG). UML 2.0 Superstructure Specification. http://www.omg.org, August 2005.

[14] Open specifications for Multi Processing (OpenMP). http://www.openmp.org/.

[15] S. Pllana, S. Benkner, F. Xhafa, and L. Barolli. Hybrid Performance Modeling and Prediction of Large-Scale Computing Systems. In 2008 International Conference on Complex, Intelligent and Software Intensive Systems (CISIS 2008), Barcelona, Spain, March 2008. IEEE Computer Society.

[16] S. Pllana, I. Brandic, and S. Benkner. A Survey of the State of the Art in Performance Modeling and Prediction of Parallel and Distributed Computing Systems. International Journal of Computational Intelligence Research (IJCIR), 4(1), January 2008.

[17] S. Pllana and T. Fahringer. On Customizing the UML for Modeling Performance-Oriented Applications. In $U M L$ 2002, "Model Engineering, Concepts and Tools", LNCS 2460, Dresden, Germany, Dresden, Germany, October 2002. Springer-Verlag.

[18] S. Pllana and T. Fahringer. UML Based Modeling of Performance Oriented Parallel and Distributed Applications. In Proceedings of the 2002 Winter Simulation Conference, San Diego, California, USA, December 2002. IEEE.

[19] A. Snavely, L. Carrington, N. Wolter, J. Labarta, R. Badia, and A. Purkayastha. A Framework for Performance Modeling and Prediction. In The 2002 ACM/IEEE conference on Supercomputing, Baltimore, Maryland, USA, November 2002. ACM.

[20] M. Snir, S. Otto, S. Huss-Lederman, D. Walker, and J. Dongarra. MPI: The Complete Reference. MIT Press, 1998. 